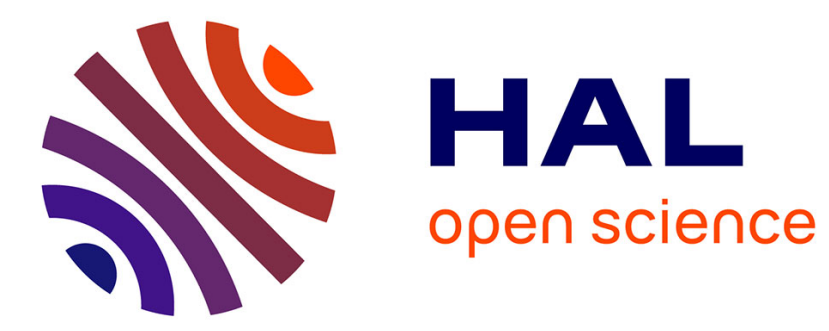

\title{
Une paternité réinventée? Le vécu parental des pères isolés
}

Agnes Martial

\section{To cite this version:}

Agnes Martial. Une paternité réinventée? Le vécu parental des pères isolés. Informations sociales, 2013, 176, pp.62-69. hal-02176582

\section{HAL Id: hal-02176582 \\ https://hal.science/hal-02176582}

Submitted on 12 Jul 2019

HAL is a multi-disciplinary open access archive for the deposit and dissemination of scientific research documents, whether they are published or not. The documents may come from teaching and research institutions in France or abroad, or from public or private research centers.
L'archive ouverte pluridisciplinaire HAL, est destinée au dépôt et à la diffusion de documents scientifiques de niveau recherche, publiés ou non, émanant des établissements d'enseignement et de recherche français ou étrangers, des laboratoires publics ou privés. 


\section{Une paternité réinventée? Le vécu parental des pères isolés}

Agnès Martial

Informations sociales, 176, 2013, p. 62-69.

\section{Résumé}

Qu'est-ce qu'être père au quotidien après un divorce ou séparation ? A partir d'une enquête menée à Marseille auprès d'une vingtaine d'hommes, et par delà la diversité des trajectoires et des appartenances sociales, le présent article interroge les traits communs de la monoparentalité masculine : poids de la charge éducative, isolement et solitude, mais aussi, pour certains, dernier ancrage social, et lieu d'invention une paternité différente.

Les ruptures conjugales transforment la paternité : le plus souvent fragilisée par les divorces et les séparations, elle peut aussi et à l'inverse être investie de manière centrale, dès lors qu'un homme est conduit à assumer seul la charge quotidienne de son ou ses enfants. Minoritaires ${ }^{\mathrm{i}}$, mal connues et peu explorées, ces situations confrontent les pères à diverses difficultés, et les amènent à reformuler leur rôle et leur statut de parent. Une enquête récente ${ }^{\mathrm{ii}}$, menée grâce à la CAF des Bouches du Rhône, nous a permis de rencontrer 24 hommes ayant connu ou connaissant une situation de paternité «en solo » à la suite d'une rupture conjugale. La diversité des trajectoires ayant été volontairement privilégiée, ces pères d'âge et d'origine culturelle variés (nés à Marseille, originaires d'autres régions françaises, migrants d'origine étrangère) étaient nantis de niveaux de qualification très différents, et appartenaient à des catégories socioprofessionnelles diversifiées (sans activité, ouvriers, employés, cadres du secteur public ou privé, indépendants).

A partir d'un tel groupe, il importe de tenir compte de la pluralité des conditions économiques et matérielles des séparations, ainsi que des variations sociales et culturelles des rapports de genre et de parenté. Cette diversité permet d'interroger les frontières établies entre un modèle dominant de séparation «négociée », allié aux principes d'égalité et de coparentalité, que la majorité des travaux sur les recompositions familiales ont décrit à partir de l'étude des classes moyennes et supérieures, et un modèle de séparation plus «traditionnel» au sein des milieux modestes ou populaires, où la rigidité plus grande des normes de genre freinerait l'investissement égalitaire et conjugué des rôles parentaux, fragilisant notamment la paternité.

\section{L’entrée en «monoparentalité » : entre discours égalitaire et disqualification de la maternité}

Différents processus ont conduit les pères rencontrés à élever quotidiennement leurs enfants après un 
divorce ou une séparation. Deux motifs principaux se détachent, qui peuvent dans certains cas coexister.

Le premier donne à voir cet arrangement comme le prolongement d'une répartition des rôles où le père occupait déjà une position de «parent principal » dans la prise en charge quotidienne des enfants, du fait de la dynamique «égalitaire » présente au sein du couple parental ou (et) du peu d'intérêt de la mère pour cette dimension de la parentalité. Les hommes témoignant de cette expérience appartiennent plutôt aux classes moyennes ou supérieures. Ils se disent déterminés à demeurer pères au quotidien, et ont demandé, dès l'annonce de la rupture, que la résidence des enfants soit fixée chez eux. Ils se présentent ainsi comme les garants d'une continuité familiale bénéfique pour les enfants. On voit se dessiner dans certains cas une situation symétriquement inverse aux répartitions « traditionnelles » des rôles de genre. Julien, 49 ans, et son ex-épouse sont tous deux architectes, parents de deux enfants. Au fil de leurs trajectoires professionnelles, la mère s'est investie de manière grandissante dans sa carrière professionnelle, tandis qu'il se consacrait plus qu'elle à l'éducation de leurs enfants, âgés de 7 et 10 ans au moment de leur séparation. Il lui a paru logique et cohérent, lorsqu'elle l'a quitté, que les enfants restent avec lui au quotidien, ce que leur mère n'a d'ailleurs pas contesté en acceptant un droit de visite et d'hébergement.

Pour certains de ces pères, cette répartition se justifie aussi par une incapacité relative de la mère à prendre en charge les enfants, second déterminant de la résidence quotidienne des enfants chez le père. Le registre de la défaillance maternelle affleure alors dans les récits. Quelque soit leur milieu d'origine, et bien que les motifs et critères de leurs jugements varient grandement, nombre de pères s'accordent à constater que les mères de leurs enfants ne sont pas - ou pas tout à fait - de «bonnes » mères. Dans les cas les plus ordinaires, la maternité est décrite comme peu investie, ou caractérisée par diverses formes d'incompétence domestique ou éducative. Les mères sont parfois présentées comme peu fiables, en raison de leur inconstance amoureuse et sexuelle, de leur instabilité professionnelle, ou (et) d'une grande fragilité psychologique. Dans le parcours de certains pères, plus fréquemment issus de milieux modestes, la mère s'était vue confiée la résidence habituelle lors de la rupture, ou devait accueillir les enfants en résidence alternée, mais cette solution n'a pas duré, pour des raisons économiques et matérielles (perte du logement), de santé (dépressions, hospitalisations), ou parce qu'un conflit mère-enfant a provoqué un changement de résidence habituelle. Dans quelques situations, la mère s'est éloignée ou a disparu, quittant à la fois les enfants et le père. Dans des cas plus rares encore, les enfants dont la résidence avait été fixée au domicile maternel ont été placés en raison de graves défaillances éducatives, le père récupérant finalement la «garde » des enfants. Au fil des récits se dessinent ainsi des paternités construites en miroir d'une maternité fragile, affaiblie ou déficiente, parfois désertée. S'il est évident que certaines de ces trajectoires renvoient à de très lourdes problématiques sociales et psychologiques, le caractère transversal de ce discours témoigne aussi de la puissance des normes définissant les conditions habituelles d'exercice de la maternité et de la 
paternité. Elles retentissent également dans l'inquiétude que ces hommes expriment fréquemment au sujet du bien-être de leurs enfants, craignant de ne pouvoir compenser l'éloignement de la mère. Loin de remettre en cause la prééminence maternelle dans le quotidien des enfants, nombre d'entre eux pallient son absence, de manière plus ou moins contrainte. Ceci n'en transforme pas moins leur manière d'agir et de se penser comme pères, et modifie les dynamiques articulant paternité et maternité dans l'après-rupture.

\section{Des familles « patricentrées »}

Sur bien des points, l'expérience masculine et solitaire de la parentalité fait écho à celle des familles monoparentales féminines. Le temps et l'espace y sont saturés d'enfants, de taches éducatives et de travail domestique, qu'il faut concilier avec un emploi assurant un revenu plus ou moins élevé, et pour lesquels il faut aussi parfois renoncer à une vie sociale qui s'amenuise ou disparaît.

Les inégalités socio-économiques sont ici très présentes. Presque tous les pères rencontrés ont du adapter leur vie professionnelle aux nouvelles contraintes familiales, ce qui semble plus aisé pour les cadres, les ingénieurs et certains indépendants que pour les petits employés ou les hommes travaillant grâce à des contrats à durée déterminée, dont ils ne peuvent négocier les horaires, souvent «atypiques » et inconciliables avec la présence au foyer de jeunes enfants. Si certains pères sont en mesure de financer la garde des enfants à domicile, d'autres dépendent entièrement des structures institutionnelles de prise en charge de la petite enfance. Ils renoncent parfois à l'emploi, et perçoivent le RSA en attendant que les enfants grandissent. Se pose également la question de la contribution de l'autre parent à l'éducation et à l'entretien de l'enfant: lorsqu'elles en ont les moyens et qu'une résidence habituelle au domicile paternel a été décidée, les mères versent une pension au père, ce qui advient dans les classes moyennes et supérieures, jamais dans les milieux modestes. Il faut enfin souligner l'importance du soutien familial, très inégal selon les situations : là où certains pères bénéficient d'une aide et d'une présence très régulière, assurée par les grands-parents ou par une sœur demeurée célibataire, d'autres, notamment les plus précaires et les migrants, demeurent très isolés.

L'investissement de la paternité comme relation quotidienne se traduit par des gestes nouveaux, au fil de la vie domestique et matérielle, par des préoccupations éducatives envahissantes, et par des échanges plus intenses avec les enfants. Ces pères sont concernés par le suivi scolaire et les relations à l'école, par la santé, l'organisation des loisirs, des vacances, etc... Ils regrettent fréquemment l'absence d'un réseau de pairs avec qui partager les difficultés ou échanger de menus services : les pères seuls se lient plus rarement aux autres parents « quotidiens », majoritairement féminins, des autres enfants. Ils expriment leur angoisse et leur sentiment de solitude lorsque leurs enfants, surtout à l'adolescence, rencontrent différentes difficultés scolaires, sociales et psychologiques. Mais ils commentent aussi l'intensité de leur relation à l'enfant et leur plaisir à partager avec lui la vie de tous les jours. La présence de la mère et son éventuelle intervention dans la vie de l'enfant sont peu mentionnées 
spontanément : ces hommes valorisent la dimension solitaire de leur paternité.

A cette charge parentale répond en effet une forme de prééminence éducative, plus ou moins explicitement affirmée. En fonction des situations, de la capacité de la mère à participer aux décisions, et de la qualité des relations entre les parents, une gamme étendue déploie différentes interprétations d'une coparentalité plus ou moins réalisée : certains pères prennent entièrement seuls les décisions relatives aux enfants, d'autres se contentent de «prévenir » la mère, d'autres encore la consultent et se déterminent en accord avec elle.

Au-delà des décisions relatives aux grandes orientations de la vie de l'enfant, cette prééminence se dessine à travers certaines manières de faire ou de dire, qui laissent deviner une position d'autorité ou de responsabilité au sein du duo parental. Medhi, 38 ans, est arrivé d'Algérie il y a 10 ans. Il est employé en CDD à temps partiel dans une entreprise de nettoyage. Marié puis divorcé d'avec une jeune femme française, il s'est rapidement occupé de son fils (9 ans) au quotidien, son ex-épouse ne parvenant pas à assumer le système d'alternance initialement fixé. L'enfant se rend néanmoins en visite chez sa mère de temps en temps. Medhi veille alors au bon déroulement de ces séjours en donnant à la mère une liste de recommandations : heure du coucher, horaires de télévision, etc., qu'elle est censée observer. Il se préoccupe également de la tenue de l'enfant, achetant des vêtements que l'enfant emmène au domicile maternel, car il juge qu'elle « sait pas marier les choses, par exemple la veste avec le pantalon, elle l'envoie \{à l'école\} n'importe comment, j'aime pas ça. C'est pas de la marque, mais bien habillé, propre. Et bien assorti. ”

Certains pères racontent avoir suggéré à la mère différentes initiatives censées entretenir ou améliorer sa relation à l'enfant, comme lui acheter, certains mois, quelques vêtements au lieu de verser la pension, ou venir veiller un enfant hospitalisé. De manière plus ou moins explicite, ils jouent ainsi un rôle de médiation dans la définition même de la relation de la mère à l'enfant. Ainsi, la coparentalité s'efface t'elle au profit de la prééminence paternelle.

\section{Le coût de la paternité quotidienne : pas ou peu de nouvelles unions}

Ce rôle de parent principal induit toutefois un coût particulièrement apparent dans ce groupe de pères $^{\mathrm{iii}}$, qui déroge aux tendances statistiques selon lesquelles les hommes se remettraient en couple plus vite et plus fréquemment que les femmes. Certains n'ont aucune vie amoureuse, se sentant trop isolés, indisponibles, dans une situation qui ne leur permet pas de rencontrer un nouveau partenaire. D'autres conjuguent un état de célibataire à quelques aventures, voire une relation régulière hors de la vie familiale, comme si leurs enfants et leurs amours devaient constituer deux univers affectifs distincts et plus ou moins cachés l'un à l'autre. Quelques uns décrivent quelques tentatives insatisfaisantes de vie commune, déçus par un premier essai qui a créé, disent-ils, des « complications » avec les enfants : tant que ces derniers ne sont pas autonomes, la relation paternelle 
passe avant la relation conjugale. Cet état de célibataire suscite parfois un discours sacrificiel : "les enfants passent d'abord, moi après ", "c'est fini pour moi, maintenant c'est tout pour mes enfants 》, " j'ai tout donné pour eux pendant six ans ». Mais au « sacrifice » que représente la paternité répond une forme d'inscription sociale parfois précieuse.

\section{La paternité comme dernier ancrage?}

Pour certains de ces pères, la paternité n'est pas seulement redéfinie comme relation nourricière et quotidienne, devenue centrale dans la configuration familiale post-rupture. Elle est aussi garante d'une identité sociale légitime, notamment quand l'échec de l'insertion professionnelle ou(et) l'expérience migratoire placent ces hommes en situation de grande fragilité. Le statut de père et sa reconnaissance se jouent d'une part dans la relation aux institutions et plus particulièrement aux services de l'aide sociale. Le versement des prestations sociales et familiales inscrit ainsi les hommes dans certaines catégories, de nouvelles dénominations apparaissant parfois : "J'ai un RSA "père isolé ", dit par exemple Medhi. Au-delà des seules prestations financières, certains hommes très isolés, débordés par la charge éducative et les difficultés de leurs enfants sont encadrés et soutenus, en tant que pères, par un dense réseau constitué des services sociaux, médico-psychologiques, parfois judiciaires, unique mode d'insertion sociale qui leur soit encore accessible. L'importance de cette reconnaissance institutionnelle se lit aussi dans les plaintes récurrentes de nombreux hommes moins précarisés, mais qui estiment que leur situation ne suscite que trop peu d'intérêt et de soutien de la part des services sociaux, ce qui traduit selon eux l'illégitimité de leur situation de père seul.

Dans l'environnement social et relationnel immédiat, la paternité peut aussi être présentée comme un motif de fierté et de réussite. Un seul père en situation précaire nous a dit son refus de faire connaitre sa situation, ressentie comme honteuse et socialement dégradante. Les autres tendent plutôt à valoriser leur paternité singulière. Mario, brésilien, travaillait comme charpentier en Guyane française quand sa femme l'a quitté, et s'est retrouvé seul avec 4 jeunes enfants. Il a cessé de travailler en arrivant en métropole, et perçoit le RSA. Il est devenu père d'un cinquième enfant à Marseille, au cours d'une relation conjugale aujourd'hui rompue. La résidence habituelle de la petite fille est fixée chez la mère, mais c'est chez lui, dit-il, qu'elle passe la majorité du temps. La paternité quotidienne est ainsi devenue une dimension centrale et valorisante de son identité sociale, assurant son insertion dans un réseau de relations d'entraides : le jour de notre rencontre, Mario est à la maison avec 3 de ses enfants, et garde aussi le petit garçon de l'une de ses amies. "C'est ça... j'étais charpentier, maçon, et maintenant je suis ... C'était pas aussi difficile (rires !) Maintenant je suis la maman, je suis le papa, les gens (...) ils disent non, je préfère laisser mes enfants avec toi, parce que je sais qu'ils seront bien. »

Ce statut particulier ne renvoie pas seulement aux capacités «maternantes » de ces hommes qui savent 
s'occuper des enfants. Il se construit aussi en référence à des manières plus traditionnelles de définir la paternité. Etre un bon père, c'est s'affirmer comme un homme courageux et responsable, assumant ses enfants, ce que ne manquent pas de souligner nos interlocuteurs en jugeant sévèrement leur pairs qui, dans une situation comparable, n'assument pas leur rôle parental. Abdallah, 40 ans, a divorcé de la femme qu'il a épousée en arrivant d'Algérie et s'occupe de leur fille au quotidien. Il est sans activité suite à la faillite du commerce qu'il avait monté à Marseille :

" J'ai connu des gens ici, quand ils me voient avec la petite ils me disent "vraiment vous êtes courageux", parce qu'ils ont des enfants mais ils sont pas capables de s'en occuper, ils les envoient au bled, il y en a qui sont remariés.... Mais moi quand ils me voient avec la petite ils me disent 'vraiment! T'as gardé la petite depuis qu'elle est bébé jusqu'à maintenant! »

Etre un père accompli, c'est aussi se montrer honnête et respectable. Medhi estime que son entourage porte un regard positif sur sa manière de vivre avec son fils. Il est plutôt fier de se montrer en compagnie de l'enfant, dont la présence auprès de lui garantit sa « bonne conduite».

"Il y en a qui m'ont dit «il te gène pas? "? Je dit « mais dit il me gène en quoi ? Je vais voler, je vais trafiquer ou quoi, je vais faire quoi ? Il est avec moi, il est avec moi ! »

La paternité quotidienne et solitaire concerne ainsi des hommes aux trajectoires personnelles, sociales et familiales très différentes, parfois très singulières, au point que l'on pourrait interroger la pertinence d'une analyse unifiant ces expériences en une seule et même catégorie. Différents éléments nous paraissent toutefois communs à ces vécus parentaux. Le premier relève d'un sentiment de solitude et d'isolement lié au caractère peu répandu et difficilement partageable de la paternité « en solo». Le second concerne la manière dont celle-ci met à l'épreuve les définitions traditionnelles des rôles de genre, conduisant fréquemment ces hommes à repenser leurs propres catégories. "Je suis le père, je suis la mère", disent certains, parce qu'ils s'estiment contraints de pallier l'absence de l'autre parent. D'autres revendiquent plutôt d'assumer des taches et des gestes habituellement attribués aux femmes, et redéfinissent alors le contenu de leur paternité. Pour tous, la parentalité devient à la fois un fardeau et une dimension majeure et prépondérante de l'existence, offrant aux plus fragilisés l'occasion de reconstruire une identité légitime et positive, une forme de respectabilité que n'assurent plus les autres formes d'inscriptions sociales. Bien que minoritaires, ces expériences paternelles singulières sont, dans tous les milieux, un lieu de mise en doute et de réinvention de la paternité contemporaine : elles appellent reconnaissance et soutien de la part des politiques publiques et des pratiques du travail social. 
${ }^{\mathrm{i}}$ La résidence des enfants au domicile paternel ne concerne que $8 \%$ des enfants mineurs au moment des divorces (Chaussebourg L., Carrasco V., Lermenier A., 2009, Le divorce, Rapport pour le Secrétariat général, sous-direction de la statistique, des études et de la documentation, Ministère de la Justice.). Elle représente cependant une réalité plus importante si l'on tient compte des modifications ultérieures de la résidence des enfants (voir Martial, «Des pères absents aux pères quotidiens : représentations et discours sur la paternité dans l'après-divorce » dans ce numéro).

ii Enquête menée dans le cadre du programme ANR-08-JCJC-0057-01-PATERNITES. Pères en solitaires : paternités contemporaines et nouvelles trajectoires familiales (France, Espagne).

iii Il importe bien sur de tenir compte du biais induit par la prise de contact, ces pères étant majoritairement déclarés auprès de la Caisse d'Allocation Familiale comme vivant seuls avec leurs enfants. 